\title{
CpG oligodeoxynucleotides are effective in therapy of minimal residual tumour disease after chemotherapy or surgery in a murine model of MHC class I-deficient, HPV16-associated tumours
}

\author{
MILAN REINIS, JANA SÍMOVÁ, MARIE INDROVÁ, JANA BIEBLOVÁ and JAN BUBENÍK \\ Institute of Molecular Genetics, Academy of Sciences of the Czech Republic, Prague 6, Czech Republic
}

Received December 14, 2006; Accepted January 29, 2007

\begin{abstract}
Oligodeoxynucleotides containing guanine-cytidine dimers (CpG ODN) are potent inducers of anti-tumour immune responses. In this study, we analyzed the capacity of $\mathrm{CpG}$ ODN to inhibit the growth of both MHC class I-positive and -deficient tumours after debulking the tumour mass by chemotherapy or surgery. We employed an animal model resembling human papillomavirus (HPV) 16-associated tumours. Tumour cell lines with distinct cell surface expression of the MHC class I molecules were injected into syngeneic C57BL/6 mice, and the growing tumours were either subjected to cytoreductive chemotherapy with ifosfamide derivative, CBM-4A, or surgically removed. Subsequent treatment with synthetic CpG ODN significantly blocked the growth of the recurrent tumours. Our results indicate that the therapy with $\mathrm{CpG}$ ODN can be effective for the treatment of minimal residual tumour disease of the tumours that have escaped from the immune surveillance by downmodulating the MHC class I expression.
\end{abstract}

\section{Introduction}

Synthetic unmethylated oligodeoxynucleotides containing guanine-cytidine dimers (CpG-ODN) are potent inducers of both MHC class I-restricted and -unrestricted immune responses $(1,2)$. Direct $\mathrm{CpG}$ ODN intratumoral administration has been shown to inhibit the growth of established tumours, including experimental cervical carcinomas on the murine models $(3,4)$. Different CpG ODN with distinct sequence

Correspondence to: Professor Jan Bubeník, Institute of Molecular Genetics, Academy of Sciences of the Czech Republic, Flemingovo nam. 2, 16637 Prague 6, Czech Republic

E-mail: bubenik@img.cas.cz

Abbreviations: HPV, human papillomavirus; B6, C57BL/6; CTL, cytotoxic T lymphocyte; NK, natural killer; $\mathrm{CpG}$, guanine-cytidine dimer; ODN, oligodeoxynucleotide; MK16, MK16/1/IIIABC

Key words: HPV16, minimal residual tumour disease, $\mathrm{CpG}$ oligodeoxynucleotides, MHC class I motives adjacent to the $\mathrm{CpG}$ islands are known to display distinct immunologic effects and, importantly, may differ in preferable induction of MHC class I-restricted or -unrestricted immune responses (5).

The most plausible therapeutic usage of CpG ODN is direct CpG ODN administration combined with the existing therapeutic modalities, e.g. surgery and/or chemotherapy and, moreover, $\mathrm{CpG}$ ODN can be used as adjuvants in combination with other immunotherapeutic agents. In these settings, the therapeutic effects of the $\mathrm{CpG}$ ODN treatment rely on activation of the immune system that may be suppressed due to the long-term antigen persistence or chemotherapy. The $\mathrm{CpG}$ ODN therapies of the residual disease after tumour surgery, chemotherapy or radiotherapy have been investigated in several studies (6-10). It has been shown that immunotherapy based on dendritic cells and CpG ODN is effective against tumours that were resistant to chemotherapy (11). In a mouse colon cancer model, combined immunotherapy with dendritic cells and $\mathrm{CpG}$ ODN resulted in tumour rejection and, moreover, decreased toxicity of immunotherapy (12).

Recently, we have shown, using a murine model, that intratumoral administration of $\mathrm{CpG}$ ODN inhibited the growth of MHC class I-positive as well as -deficient experimental tumours associated with HPV16 (4). This is of particular importance since the tumour cells, including cervical carcinoma, frequently escape specific immune responses mediated by CTLs by downmodulation of MHC class I surface expression (13). Thus, CpG ODN treatment may meet the requirement of effective anti-tumour immunotherapy that should act on a broad spectrum of tumours of the same aetiology but different or changing MHC class I expression (14). However, no studies have yet compared the efficacy of the CpG ODN treatment of the MHC class I-positive and -deficient tumours of the same aetiology, i.e. expressing the same tumour-associated antigens, in combination with surgery or chemotherapy.

In this study, we have investigated the efficacy of $\mathrm{CpG}$ ODN administration for the therapy of experimental minimal residual tumour disease after chemotherapy with cyclophosphamide derivative CBM-4A or surgery in consideration of MHC class I expression on the tumour cells, i.e. MHC class I positive and MHC class I-deficient tumours associated with HPV16. 


\section{al}

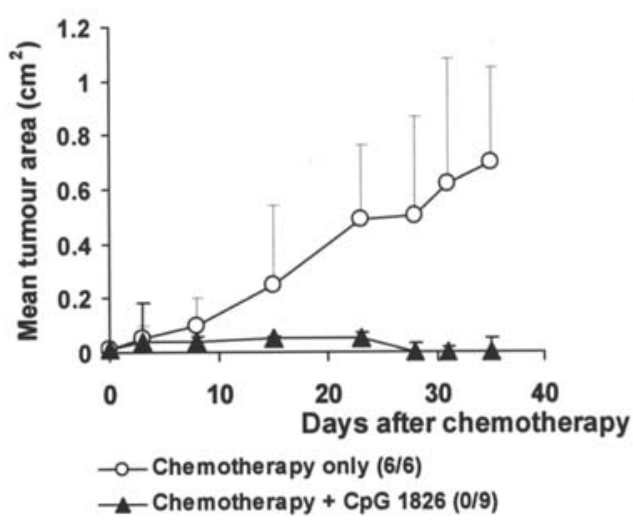

b/

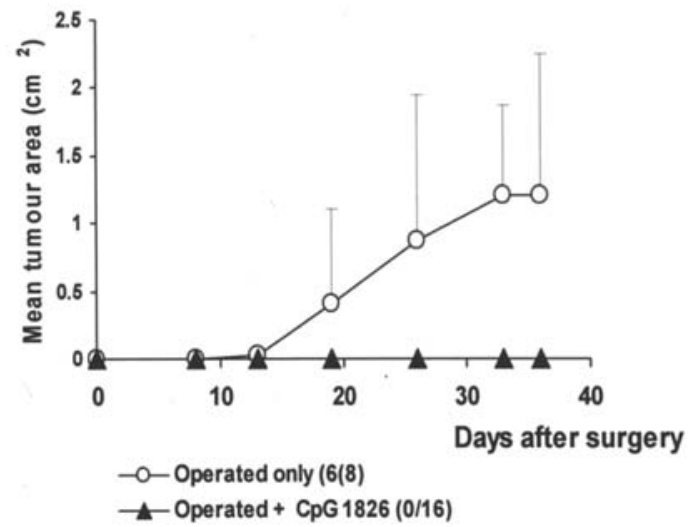

Figure 1. CpG ODN 1826 treatment prevents the growth of TC-1 (MHC class I-positive) recurrent tumours. Mice were injected with 2x10 4 TC-1 cells. Growing tumours were debulked either by cytoreductive chemotherapy (a) or surgically (b) and mice were treated with CpG ODN 1826 . Controls were mice that underwent only chemotherapy or surgery, respectively. CpG ODN 1826 treatment resulted in elimination of the recurrent tumours and CpG ODN-treated mice remained tumour-free. The numbers of tumour takes in the $\mathrm{CpG}$ ODN-treated experimental groups were significantly different as compared to the control groups (determined by the $\chi^{2}$ test, $\mathrm{p}<0.05$ ). Numbers in parentheses represent numbers of mice with tumour takes/numbers of mice in experimental groups. Experiments were performed twice with similar results.

\section{Materials and methods}

Mice. C57BL/6 (B6) male mice, 8-10 weeks old, were obtained from Anlab Co., Prague, Czech Republic. Experimental protocols were approved by the Institutional Animal Care Committee of the Institute of Molecular Genetics, Prague.

Cell lines. TC-1 cell line is MHC class I-positive, of lung origin, non-metastasizing, and immunogenic in the syngeneic C57BL/6 mice (15). This cell line expressing human E6/E7 oncogenes was prepared by co-transfection of C57BL/6 cells with HPV16 E6/E7 DNA and activated ras. TC-1/A9 is an MHC class I-negative derivative of the TC-1 cell line (16). MK16/1/IIIABC (MK16) cell line is MHC class I-negative, of kidney origin, and develops metastases (17).

Synthetic CpG ODN. CpG ODN 1826 (5-TCCATGACGTT CCTGACGTT-3) is a potent inducer of both adaptive and innate immunity and has been described to activate both CTL and NK cells $(18,19)$. CpG ODN 1585 (5-GGGGTCAA CGTTGAGGGGGG-3) has been optimized for NK activation (20). Both CpG ODN, purchased from Generi Biotech, Czech Republic, were sulphur-modified in their backbone (phosphorothioate) and synthesized under endotoxin-free conditions.

Chemotherapy. Mice were inoculated s.c. with tumour cells. When the tumours reached a size of 2-3 mm in diameter, mice were injected i.p. with $150 \mathrm{mg} / \mathrm{kg}$ of bromine-substituted derivative of ifosfamide CBM-4A (racemic chlorobromofosfamide) preparation $(21,22)$. The CBM-4A treatment was designed to inhibit but not completely eliminate the growth of the tumours.

Surgical treatment. Mice were inoculated s.c. with tumour cells. Approximately 30 days later, when the transplanted tumours reached $\sim 8-12 \mathrm{~mm}$ in diameter, the tumours were excised under i.p. anaesthesia, leaving no macroscopically visible tumour residuum $(22,23)$.

CpG-ODN treatment. CpG-ODN therapy started two days after surgery or six days after cytoreductive chemotherapy (50 $\mu \mathrm{g}$ in $50 \mu \mathrm{l}$ PBS per dose), $\mathrm{CpG}$ ODN were injected directly into the tumours or into the site of the previous operation or to the tumour cell injection site if the tumours were not palpable. Two doses were injected in the first week of the treatment and the treatment continued with 1 injection/ week. The experimental and control mice were observed bi-weekly and the number of tumour takes was recorded. On tumour-bearing animals, two perpendicular diameters of the tumours were measured with a calliper and the tumour size was expressed as the tumour area $\left(\mathrm{cm}^{2}\right)$.

Statistical analyses. For statistical analyses of differences between the growth curves of tumours, the analysis of variance from NCSS, Number Cruncher Statistical System (Kaysville, UT), statistical package was used. For comparison of tumour takes in experimental and control groups, the $\chi^{2}$ test was utilized.

\section{Results}

CpG ODN 1826 treatment prevented the growth of MHC class I-positive and-deficient recurrent tumours. The capability of CpG ODN 1826 to prevent the growth of recurrent tumours after surgery or to cure minimal residual tumour disease after chemotherapy was investigated and compared. In both settings, CpG ODN 1826 treatment resulted in complete elimination of the recurrent TC- 1 tumours and on day $35 \mathrm{CpG}$ ODN-treated mice remained tumour-free or regressed, respectively (Fig. 1). To assess the efficacy of the CpG ODN 1826 treatment of the TC-1 MHC class I-negative counterparts of the TC- 1 tumours, the experiments were 
al

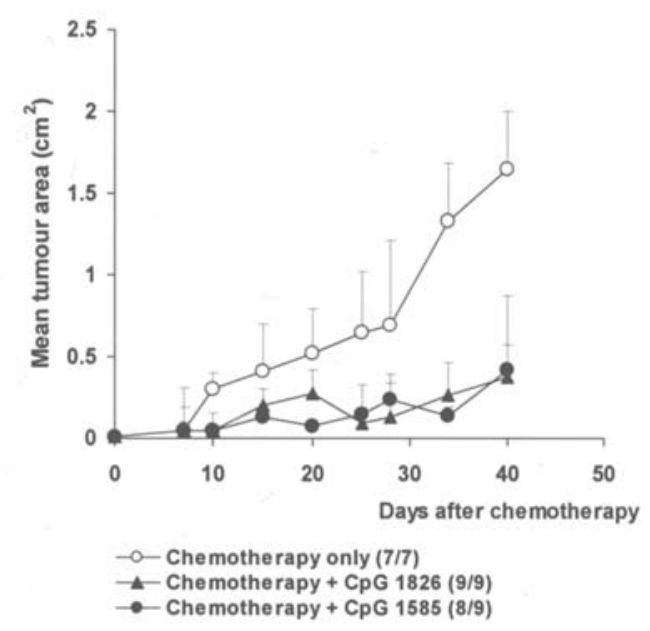

$\mathrm{b} /$

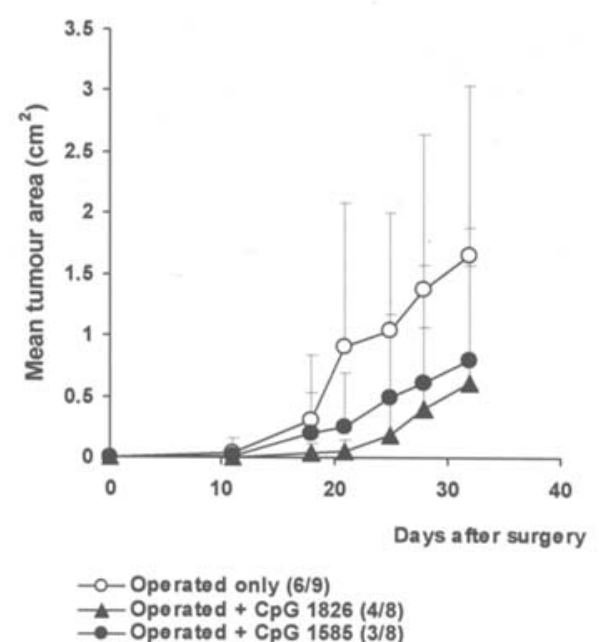

Figure 2. CpG ODN 1826 and 1585 treatment inhibits the growth of TC-1/A9 (MHC class I-negative) recurrent tumours. Mice were injected with 2x104 TC-1/ A9 cells. Growing tumours were debulked either by cytoreductive chemotherapy (a) or surgically (b) and mice were treated with CpG ODN 1826 or CpG ODN 1585. Controls were mice that underwent only chemotherapy or surgery, respectively. Significant differences in the tumour size (determined by Newman-Keels and Tukey-Kramer tests, $\mathrm{p}<0.05$ ) were observed between the $\mathrm{CpG}$ ODN-treated mice and control mice. No significant differences were observed between CpG ODN 1826- and CpG ODN 1585-treated mice. Numbers in parentheses represent numbers of mice with tumour takes/numbers of mice in experimental groups. Experiments were performed twice with similar results.

al

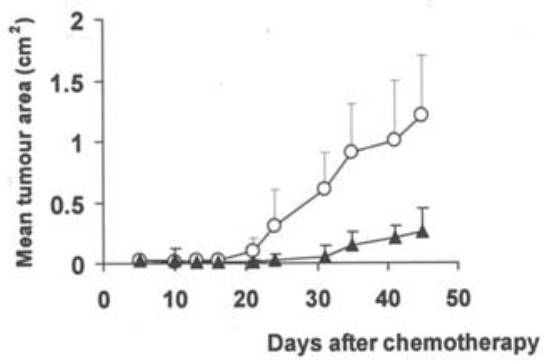

- - Chemotherapy only (7/9)

$\rightarrow$ Chemotherapy + CpG $1826(2 / 9)$ $\mathrm{b} /$

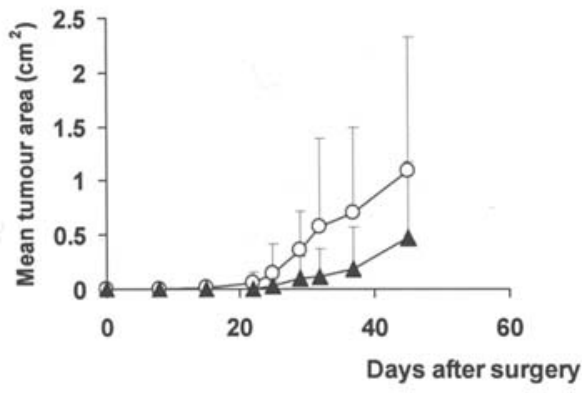

$-0-$ Operated only (8/9)

- Operated + CpG $1826(7 / 8)$

Figure 3. CpG ODN 1826 treatment inhibits the growth of MK16 (MHC class I-deficient) recurrent tumours. Mice were injected with $1 \times 10^{5}$ MK16 cells. Growing tumours were debulked either by cytoreductive chemotherapy (a) or surgically (b) and mice were treated with CpG ODN 1826 . Controls were mice that underwent only chemotherapy or surgery, respectively. Significant differences in the tumour size (determined by Newman-Keels and Tukey-Kramer tests, $\mathrm{p}<0.05$ ) were observed between the $\mathrm{CpG}$ ODN-treated mice and control mice. Numbers in parentheses represent numbers of mice with tumour takes/ numbers of mice in experimental groups. Experiments were performed twice with similar results.

performed with the TC-1/A9 tumour cell line. In these experiments, the differences in the tumour size were observed between the $\mathrm{CpG}$ ODN-treated mice and control mice (Fig. 2). However, unlike TC-1 tumours, the mice were not tumourfree after the treatments.

CpG ODN 1585 treatment inhibits the growth of TC-1/A9 (MHC class I-deficient) recurrent tumours. We made a comparison of CpG ODN 1826 and 1585, two ODN capable of coping with MHC class I-deficient tumours (Fig. 2). Growing tumours were debulked either by chemotherapy or surgically and mice were treated with CpG ODN 1585 that has been previously described to be effective in monotherapy against TC-1/A9 but not against TC-1 tumours (4). The results revealed that there were no significant differences between CpG ODN 1826- and CpG ODN 1585-treated mice and both CpG ODN inhibited the TC-1/A9 tumour growth.

CpG ODN 1826 treatment inhibits the growth of MK16 (MHC class I-deficient) recurrent tumours. Further, we compared the effectiveness of CpG ODN immunotherapy of minimal residual tumour disease between two E6- and E7-expressing tumours TC-1/A9 and MK16, which were both phenotypically MHC class I-negative due to impaired APM, but of different origin. The experiments showed that the efficacy of the treatments after chemotherapy and surgery were similar 
(Figs. 2 and 3). As in experiments on TC-1/A9, the growth of recurrent or residual MK16 tumour was inhibited but not completely prevented.

\section{Discussion}

This study inferred a strong potential of synthetic CpG ODN for the therapy of the minimal residual tumour disease of both MHC class I-positive and -deficient, HPV16-associated tumours. We have demonstrated, using two distinct MHC class I-deficient tumour cell lines, that two ODN harbouring different $\mathrm{CpG}$ motives were effective in the treatment of minimal residual tumour disease, albeit the efficacy was lower than in the control experiment on MHC class I-positive tumours.

We have used a model in which the MHC class I downregulation was reversible and resulted from impaired antigenprocessing machinery (APM). This is of clinical relevance since the MHC class I-deficient tumour derivatives originating from coordinated downregulation of APM components are frequently observed in HPV-associated cervical carcinomas in humans $(24,25)$. In our previous experiments, we have shown that effective immune responses against MHC class I-deficient tumour cells due to APM defects are mediated mainly by $\mathrm{NK} 1.1^{+}$cells and not by $\mathrm{CD} 4^{+}$or $\mathrm{CD}^{+}$cells. These findings indicate that the tumours are phenotypically MHC class Inegative and, moreover, they are not targeted by CTLs after possible MHC class I upregulation during tumour development, e.g. due to cytokine effects (4).

Current therapeutic experimental protocols based on the CpG ODN treatment are frequently based on combination therapies with surgery or chemotherapy. Effective treatments of minimal residual tumour disease with $\mathrm{CpG}$ ODN, either alone or in combination with specific vaccines, have been described and $\mathrm{CpG}$ ODN is a powerful tool for activation of the immune system and, notably, for reversal of immunosuppression $(6,8)$. It has been well documented that immunosuppression induced by tumour growth or anti-tumour chemotherapy is reversible and the immune system can become functional after tumour excision or termination of chemotherapy. The rate of this recovery is probably of particular importance for prevention of the tumour recurrences, and combination therapy with immunostimulators, e.g. $\mathrm{CpG}$ ODN treatment, can accelerate this process.

So far, there is a lack of information on the CpG ODN therapy of minimal residual tumour disease when the tumours have escaped the CTL-mediated immune response by downmodulating MHC class I expression. Recently we have shown, using the same experimental model as in this study, that synthetic $\mathrm{CpG}$ containing oligodeoxynucleotides have a potential for the therapy of HPV16-associated tumours regardless of their MHC class I status (4). Thus, a question to be addressed was whether $\mathrm{CpG}$ ODN could also cope with a minimal residual tumour disease of the MHC class Ideficient tumours. The results of this study indicate that both CpG ODN 1826 and CpG ODN 1585, which have been optimized for NK activation, can inhibit the growth of MHC class I-deficient tumours. However, the treatment only inhibited the tumour growth but did not prevent the growth of recurrent tumours or induce complete tumour regression, as compared to the treatment of parental, MHC class I-positive, TC-1 cells. These results therefore suggest that $\mathrm{CpG}$ ODN treatment is effective in the treatment of minimal residual tumour disease of the MHC class I-deficient tumours but should be combined with further treatments. An attractive speculation is that combination of chemotherapy with the CpG ODN treatment could result in lower doses of the chemotherapeutic agents and thus in less dramatic adverse effects of the therapy.

In conclusion, $\mathrm{CpG}$ ODN treatment can be used for therapy of minimal residual tumour disease of the tumours with unknown MHC class I status. This is of particular importance since MHC class I expression may be distinct on primary vs. recurrent tumours. The efficacy of the direct intratumoral (or into the site of the ablated tumour) $\mathrm{CpG}$ ODN administration after surgery or chemotherapy suggests that CpG ODN treatment of the MHC class I-deficient tumours can be effective in combination with chemotherapy or surgery in the potentially immunosuppressed environment due to the longterm antigen persistence or immunosuppressive effects of the chemotherapeutic agents.

\section{Acknowledgements}

We are grateful to Dr T.C. Wu, who kindly provided TC-1 cells, to Dr M. Smahel for TC-1/A9 cells, Professor V. Vonka for MK16 cells and to Dr E. Pajtasz-Piasecka for CBM-4A. This study was supported by grants No. 301/04/0492 and No. 301/06/0774 from the Grant Agency of the Czech Republic, by the grant from the League Against Cancer, Prague, by grant No. AVOZ50520514 from the Academy of Sciences of the Czech Republic and by EU-FP6-NoE Clinigene Project No. 018933. This study was presented in part at the 23rd IPV conference, Prague 2006.

\section{References}

1. Ballas ZK, Rasmussen WL and Krieg AM: Induction of NK activity in murine and human cells by $\mathrm{CpG}$ motifs in oligodeoxynucleotides and bacterial DNA. J Immunol 157: 1840-1845, 1996.

2. Krieg AM: CpG motifs: the active ingredient in bacterial extracts? Nat Med 9: 831-835, 2003.

3. Baines $\mathbf{J}$ and Celis $\mathbf{E}$ : Immune-mediated tumor regression induced by CpG-containing oligodeoxynucleotides. Clin Cancer Res 9: 2693-2700, 2003.

4. Reinis M, Simova J and Bubenik J: Inhibitory effects of unmethylated $\mathrm{CpG}$ oligodeoxynucleotides on MHC class Ideficient and -proficient HPV16-associated tumours. Int J Cancer 118: 1836-1842, 2006.

5. Ballas ZK, Krieg AM, Warren T, Rasmussen W, Davis HL, Waldschmidt V and Weiner GJ: Divergent therapeutic and immunologic effects of oligodeoxynucleotides with distinct CpG motifs. J Immunol 167: 4878-4886, 2001.

6. Weigel BJ, Rodeberg DA, Krieg AM and Blazar BR: CpG oligodeoxynucleotides potentiate the antitumor effects of chemotherapy or tumor resection in an orthotopic murine model of rhabdomyosarcoma. Clin Cancer Res 9: 3105-3114, 2003.

7. Milas L, Mason KA, Ariga H, Hunter N, Neal R, Valdecanas D, Krieg AM and Whisnant JK: CpG oligodeoxynucleotide enhances tumor response to radiation. Cancer Res 64: 5074-5077, 2004.

8. Wang XS, Sheng Z, Ruan YB, Guang Y and Yang ML: CpG oligodeoxynucleotides inhibit tumor growth and reverse the immunosuppression caused by the therapy with 5 -fluorouracil in murine hepatoma. World J Gastroenterol 11: 1220-1224, 2005.

9. Meng Y, Carpentier AF, Chen L, Boisserie G, Simon JM, Mazeron JJ and Delattre JY: Successful combination of local $\mathrm{CpG}-\mathrm{ODN}$ and radiotherapy in malignant glioma. Int J Cancer 116: 992-997, 2005 . 
10. Mason KA, Neal R, Hunter N, Ariga H, Ang K and Milas L: $\mathrm{CpG}$ oligodeoxynucleotides are potent enhancers of radio- and chemoresponses of murine tumors. Radiother Oncol 80: 192-198, 2006.

11. Heckelsmiller K, Beck S, Rall K, Sipos B, Schlamp A, Tuma E, Rothenfusser S, Endres S and Hartmann G: Combined dendritic cell- and $\mathrm{CpG}$ oligonucleotide-based immune therapy cures large murine tumors that resist chemotherapy. Eur J Immunol 32: 3235-3245, 2002.

12. Bourquin C, Schreiber S, Beck S, Hartmann G and Endres S: Immunotherapy with dendritic cells and $\mathrm{CpG}$ oligonucleotides can be combined with chemotherapy without loss of efficacy in a mouse model of colon cancer. Int J Cancer 118: 2790-2795, 2006.

13. Koopman LA, Corver WE, van der Slik AR, Giphart MJ and Fleuren GJ: Multiple genetic alterations cause frequent and heterogeneous human histocompatibility leukocyte antigen class I loss in cervical cancer. J Exp Med 191: 961-976, 2000.

14. Bubenik J and Vonka V: MHC class I status of tumours and design of immunotherapeutic strategies. Immunol Lett 90: 177-178, 2003

15. Lin KY, Guarwieri FG, Staveley O'Carroll KF, Levitsky HJ, August JT, Pardoll DM and Wu TC: Treatment of established tumours with a novel vaccine that enhances major histocompatibility class II presentation of tumour antigen. Cancer Res 56: 21-26, 1996.

16. Smahel M, Sima P, Ludvikova V, Marinov I, Pokorna D and Vonka V: Immunisation with modified HPV16 E7 genes against mouse oncogenic TC-1 cell sublines with downregulated expression of MHC class I molecules. Vaccine 21: 1125-1136, 2003.

17. Smahel M, Sobotkova E, Bubenik J, Simova J, Zak R, Ludvikova V, Hajkova R, Kovarik J, Jelinek F, Povysil C, Marinov J and Vonka V: Metastatic MHC class I-negative mouse cells derived by transformation with human papillomavirus type 16. Br J Cancer 84: 374-380, 2001.
18. Yi AK and Krieg AM: CpG DNA rescue from anti-IgM-induced WEHI-231 B lymphoma apoptosis via modulation of I kappa B alpha and I kappa B beta and sustained activation of nuclear factor-B/c-Rel. J Immunol 160: 1240-1245, 1998.

19. Miconnet I, Koenig S, Speiser D, Krieg A, Guillaume P, Cerottini JC and Romero P: CpG are efficient adjuvants for specific CTL induction against tumor antigen-derived peptide. J Immunol 168: 1212-1218, 2002.

20. Gramzinski RA, Doolan DL, Sedegah M, Davis HL, Krieg AM and Hoffman SL: Interleukin-12- and interferon-dependent protection against malaria conferred by $\mathrm{CpG}$ oligodeoxynucleotide in mice. Infect Immun 69: 1643-1649, 2001.

21. Indrova M, Bubenik J, Simova J, Bieblova J, Jandlova T, Smahel M, Glazman-Kusnierczyk H, Pajtasz-Piasecka E, Vonka V, Radzikowski C and Mikysková R: Chemoimmunotherapy of cancer: Potentiated effectiveness of granulocytemacrophage colony-stimulating factor and ifosfamide derivative CBM-4A. Oncol Rep 8: 1371-1374, 2001.

22. Mikyskova R, Indrova M, Simova J, Jandlova T, Bieblova J, Jinoch P, Bubenik J and Vonka V: Treatment of minimal residual disease after surgery or chemotherapy in mice carrying HPV16associated tumours: Cytokine and gene therapy with IL-2 and GM-CSF. Int J Oncol 24: 161-167, 2004.

23. Reinis M, Indrova M, Mendoza L, Mikyskova R, Bieblova J, Bubenik J and Simova J: HPV16-associated tumours: therapy of surgical minimal residual disease with dendritic cell-based vaccines. Int J Oncol 25: 1165-1170, 2004.

24. Cromme FV, Airey J, Heemels MT, Ploegh HL, Keating PJ, Stern PL, Meijer CJ and Walboomers JM: Loss of transporter protein, encoded by the TAP-1 gene, is highly correlated with loss of HLA expression in cervical carcinomas. J Exp Med 179: 335-340, 1994.

25. Keating PJ, Cromme FV, Duggan-Keen M, Snijders PJ, Walboomers JM, Hunter RD, Dyer PA and Stern PL: Frequency of down-regulation of individual HLA-A and -B alleles in cervical carcinomas in relation to TAP-1 expression. Br J Cancer 72: 405-411, 1995. 